\title{
Deleuze, Guattari et le posthumanisme
}

Alain Beaulieu

Même si le terme «posthumanisme» est absent des travaux de Deleuze et Guattari, leurs outils conceptuels ont des ressources permettant de prendre part à certains débats entourant le posthumanisme. Deleuze et Guattari n'ont jamais cessé de problématiser les conceptions traditionnelles de l'être humain. La raison comme faculté supérieure de connaissance de la vérité devient pour eux une capacité immanente d'identifier les problèmes réels auxquels ne correspondent plus aucune réponse simple, les émotions subjectives sont secondarisées par rapport aux affects et aux percepts impersonnels, et le corps dévalorisé par la tradition idéaliste devient "corps sans organes» en mesure d'expérimenter et d'exprimer les intensités vitales. En outre, la vision non-anthropocentrique de Deleuze et Guattari est librement inspirée, notamment, des potentia de la nature chez Spinoza, ainsi que du vitalisme nietzschéen. Toutefois, Deleuze et Guattari deviennent plus originaux en laissant une place à un «sujet minimal» doté d'une certaine dose de conscience, de raison et d'émotions comme autant de moyens de protection vis-à-vis du pur chaos. Tout juste ce qu'il faut de ces attributs humains demeure nécessaire afin que le «sujet» puisse naviguer dans le monde en évitant, toutefois, l'illusion d'en être maître et possesseur. Le sujet minimal, qui subit plusieurs variations notionnelles dans les travaux de Deleuze et Guattari (moi dissous, uje» divisé ou fracturé, «superject», etc.), apporte une lucidité dans un chaosmos non-familier. Situer le sujet minimal au milieu de processus de désubjectivation, et dans un monde semi-organisé, constitue d'ailleurs une clé de lecture qui permet d'apprécier la nouveauté de la critique deleuzo-guattarienne de la division sujet/objet, en en particulier de l'humanisme.

Sans prétendre épuiser la question du rapport de Deleuze et Guattari au posthumanisme, nous souhaitons présenter brièvement trois aspects de leur pensée corrélatifs de leur critique de la subjectivité et qui interfèrent avec les débats posthumanistes: les machines, les devenirs non-humains de l'homme et les forces cosmiques.

Deleuze et Guattari ne sont pas effrayés outre mesure par le monde technologique, au point d'intégrer les «machines» comme concept à part entière dans leur philosophie (machines désirantes, machines de guerre, machines abstraites, assemblages machiniques, etc.). Les machines n'ont pas pleine conscience, elles sont fabriquées pour produire, l'éventuel dysfonctionnement fait partie de leur normalité, et elles sont souvent associées à une machinerie plus complexe. En ce sens, les sujets minimaux sont similaires aux machines en tant qu'ils sont également en mesure de produire des agencements machiniques. L'humain pensé comme machine en un sens deleuzo-guattarien n'est donc pas une modalité inférieure d'existence en tant que l'homme-machine de Deleuze et Guattari peut combattre, sur un plan d'égalité, les appareils machiniques de capture d'État. Toutefois, toute tentative visant à associer Deleuze et Guattari avec l'idéologie transhumaniste visant une amélioration de la condition humaine par les moyens technologiques est vouée à l'échec puisque les machines de Deleuze et Guattari ne parviennent jamais à réaliser des états idéaux.

Être humain, pour Deleuze et Guattari, signifie singulièrement expérimenter des devenirs non-humains. Non pas au sens où l'homme aurait à imiter la plante 
ou l'animal, c'est-à-dire aller d'une identité "AA» à une identité «Bß, mais plutôt au sens où l'humain a la capacité d'expérimenter des forces impersonnelles de déterritorialisation qui animent les entités non-humaines dès lors que ces entités créent des agencements avec une vie inorganique indépendante qui relie toutes les créatures et les entités non-organiques. Les affects et les percepts impersonnels que rendent possibles les devenirs non-humains de l'homme jovent un rôle central dans les créations artistiques de «blocs de sensation», de même que dans les créations politiques d'un «peuple à veninı. En outre, sur le plan de la philosophie pratique, l'étude deleuzo-guattarienne des capacités du corps, ou l'étude de la meilleure façon pour les corps de s'affecter entre eux par l'intermédiaire d'une vie inorganique, ouvre sur une éthologie philosophique originale.

Dans Différence et répétition, Deleuze associe le livre de philosophie à une sorte de science-fiction ayant comme but de produire des visions non-familières de la réalité, de créer un monde inédit. Cette idée prend un tournant dans Mille plateaux où les forces cosmiques deviennent un vecteur de déterritorialisation. Le "cosmos» doit être entendu, ici, en un double sens : 1) la planète terre elle-même avec ses diverses strates géologiques et sa multitude d'organismes vivants, de même que 2) les forces de l'univers (ou du multivers) en entier conçu comme un "Dehors» ou un lointain plus lointain que toute forme d'extériorité, et de ce fait, paradoxalement, entrant dans un rapport avec le plus intime. Les forces extragalactiques font partie de unotre» monde, et elles peuvent affecter les humains, en dépit de leur distance physique. II ne serait sans doute pas faux de considérer que la philosophie de Deleuze et Guattari est la plus apte à nous préparer à une rencontre du troisième type, au grand plaisir des passionnés d'exobiophilie! Toutefois, ultimement, Deleuze et Guattari demeurent près de Nietzsche qui invitait à urester fidèle à la terreı, c'est-à-dire au potentiel de déterritorialisation de notre environnement immédiat.

La philosophie de Deleuze et Guattari est compatible avec certains aspects ou certaines tendances de la pensée posthumaniste. Elle peut même contribuer au développement de ce champ d'étude. Toutefois, cela serait une erreur que d'associer Deleuze et Guattari au transhumanisme (malgré leur intérêt pour la technologie), à l'anti-humanisme privé de considérations éthiques (malgré leur intérêt pour les devenirs non-humains de l'homme), ou à des aberrations désirantes para-humaines liées aux espaces cosmiques (malgré leur intérêt pour les forces cosmiques).

Cette esquisse, brève et incomplète, devrait contribuer à réfuter certains arguments posthumanistes qui ont été développés, ou qui pourraient l'être, par des lecteurs de Deleuze et Guattari. Elle devrait ainsi permettre d'éviter des débats stériles que des commentateurs antipathiques à Deleuze et Guattari seraient tentés d'initier.

\section{Références}

Deleuze, G. (1968). Différence et répétition. Presses universitaires de France.

Deleuze, G. \& Guattari, F. (1972). L'Anti-OEdipe. Minuit.

Deleuze, G. \& Guattari, F. (1980). Mille plateaux. Minuit.

Deleuze, G. \& Guattari, F. (1991), Qu'est-ce que la philosophie?. Minuit. 